\title{
Revisionsendoprothetik am Schultergelenk
}

\author{
Helmut Lill, Alexandra Hertel, Jan Christoph Katthagen, Christine Voigt
}

\section{Zusammenfassung}

Die Revisionsschulterendoprothetik ist anspruchsvoll und sollte Spezialisten in entsprechenden Zentren vorbehalten sein. Die genaue Analyse des Prothesenversagens und die Planung der Revisionsprothese sind unabdingbar. In der Unfallchirurgie stellt der Wechsel von Frakturprothesen auf inverse Prothesen die häufigste Indikation dar.

\section{Revision Shoulder Arthroplasty}

Revision shoulder arthroplasty is a challenging procedure and should only be performed by specialists in appropriate centres. An exact analysis of the prosthesis failure and accurate planning of the revision arthroplasty are mandatory. In trauma surgery the replacement of a fracture prosthesis by a reverse shoulder arthroplasty is the most common indication.

\section{Einleitung}

Die Revisionsschulterendoprothetik ist sehr komplex und mit deutlich schlechteren Ergebnissen im Vergleich zur primären Endoprothetik behaftet.

Eine Schmerzlinderung kann lediglich bei $60 \%$ der Patienten erreicht werden [5]. Eine Verbesserung der Bewegung und Alltagsfunktion ist bei insuffizienter bzw. nicht mehr vorhandener Rotatorenmanschette ebenso eingeschränkt. Wichtig in diesem Zusammenhang ist, darauf hinzuweisen, dass man den Patienten vor der Operation nicht zuviel versprechen darf.

Nach Gohlke und Rolf [3] sind v.a. folgende Faktoren für die Verbesserung der Funktion auf der Basis der pathologischen Ausgangssituation von Bedeutung:

- Entfernung des vorbestehenden Implantats

- sichere Verankerung des Revisionsimplantats mit Wiederherstellung der Länge des Humerus

- ggf. Rotatorenmanschettenrekonstruktion bzw. Refixation der Tubercula

OP-JOURNAL 2010; 26: 4-7

(c) Georg Thieme Verlag KG Stuttgart · New York DOI http://dx.doi.org/10.1055/s-0030-1250123
- periartikuläre Arthrolysen mit Entfernung von Verwachsungen

$\mathrm{Zu}$ berücksichtigen sind weiterhin die Zugangswege der Voroperation bzw. Voroperationen. Bevorzugt wird in der Revisionsendoprothetik der vordere Sulcusdeltoideo-pectorale Zugang mit sorgfältiger Schonung des Musculus deltoideus. Ausgeschlossen werden sollte, wenn möglich, eine sog. Low-Grade-Infektion. Die Komplikationsrate wird in einer neuen Arbeit [1] von 485 Fällen mit 11,6\% angegeben, wobei diese in 3 Kategorien unterteilt wurden:

ohne Re-Operation 7\%, Weichteilrevisionen 2,3\%, Implantatrevisionen 2,3\%. Nichtsdestoweniger geben $84 \%$ der Patienten mit inverser Revisionsendoprothetik an, dass Funktion und Schmerz deutlich besser sind im Vergleich zu vor der Revisionsoperation [2]. Immerhin werden von dieser Arbeitsgruppe 43\% intraoperative und $38 \%$ postoperative Komplikationen angegeben sowie eine sekundäre Infektionsrate von 10\%. Die Resektionsarthroplastik im Vergleich zur Revisionsprothetik stellt dabei immer nur die schlechtere Alternative dar. Im zwei- oder mehrzeitigen Vorgehen sollte bei Infektsituationen oder bei Verdacht auf einen Infekt die Situation beherrscht werden. Dabei sollte zur Erhaltung der Humeruslänge und als Platzhalter für eine neue Prothese ein temporärer, ent- sprechend geformter Spacer implantiert werden. Eine Antibiotikakettenimplantation ist auch temporär von Nachteil [8].

\section{Spezielle Prothesenwechsel- operationen}

Re-Surfacing Schulterkappe bzw. schaftfreier Kopfersatz auf Schaftprothese, ggf. mit Glenoidersatz (Abb. 1 und 2)

Diese Situationen treten auf, wenn sich die Schulterkappe bzw. der schaftlose Kopfersatz z.B. aufgrund von Osteonekrosen lockern. Der Wechsel auf eine geschaftete Prothese ist relativ einfach, da man entweder im Collum anatomicum osteotomiert bzw. bei dem Kopfersatz das Implantat entfernt und dann das standardisierte Vorgehen für eine geschaftete Prothese durchführt. Auch der Glenoidersatz ist nach Resektion am Collum anatomicum nach dem üblichen Vorgehen durchzuführen.

Gerade in der Revisionsprothetik sollte man auch den zusätzlichen Glenoidersatz durchführen, da die langfristigen Ergebnisse ohne Glenoidersatz schlechter werden [6].

In dieser Situation ist aufgrund von deutlichen Vernarbungen sowohl der Rotatorenmanschette als auch der Kapsel die Präparation und Exposition des proximalen Humerus deutlich erschwert. Gleiches gilt auch für den Glenoidersatz.

Schaft- und Pfannenwechsel bei gelockerter anatomischer/inverser Prothese

In Abhängigkeit von der Lockerung, ob es sich um den Schaft oder um die Pfanne handelt, werden die Prinzipien des Prothesenwechsels umgesetzt. Bei gelockerten Schäften kann dieser in der Regel ausgeschlagen und ein Revisionsschaft implantiert werden (zementiert/nicht zementiert). Sollte sich der Schaft trotz Lockerungszeichen nicht ausschlagen lassen, so muss die Schaftosteotomie in 

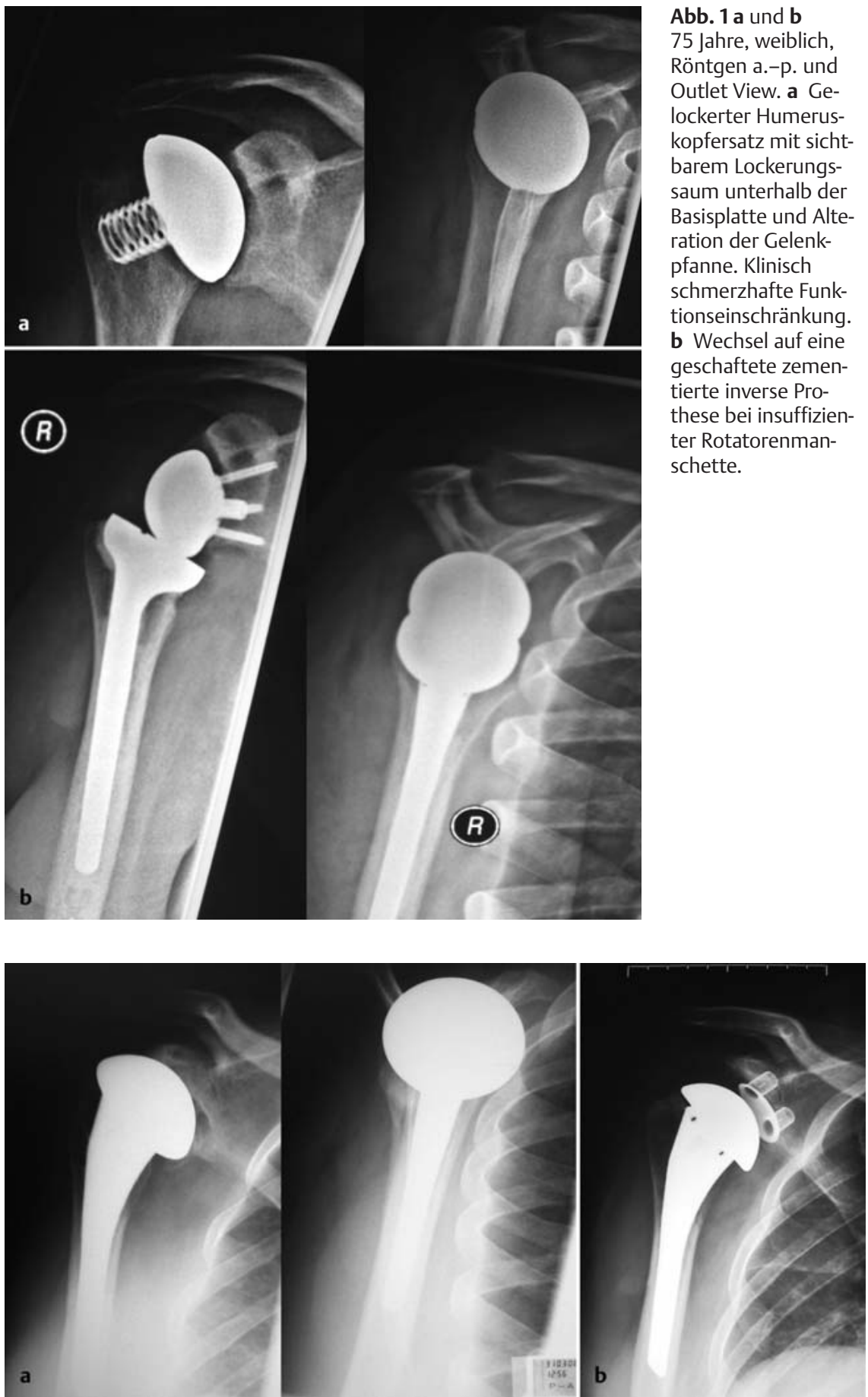

Abb. 2 a und b 46 Jahre, weiblich. a Röntgen a.-p. und Outlet View: sekundäre glenoidale Arthrose bei primärem isolierten Humeruskopfersatz. Klinisch schmerzhafte Funktionseinschränkung. b Röntgen a.-p.: sekundärer zementfreier Pfannenersatz. unten beschriebener Weise durchgeführt werden. Zur Anwendung kommen in der Regel Revisionsprothesen mit Langschaftkomponente, ggf. modulare Systeme. Der Pfannenwechsel kann sich schwieriger darstellen, da auch hier Substanzdefekte der Pfanne vorliegen. Ggf. muss ein Aufbau, z. B. mit einem Beckenkammspan, durchgeführt und dann
Abb. 1 a und $b$ 75 Jahre, weiblich, Röntgen a.-p. und Outlet View. a Gelockerter Humeruskopfersatz mit sichtbarem Lockerungssaum unterhalb der Basisplatte und Alteration der Gelenkpfanne. Klinisch schmerzhafte Funktionseinschränkung. b Wechsel auf eine geschaftete zementierte inverse Prothese bei insuffizienter Rotatorenmanschette.
Resorption der Tubercula bzw. die Insuffizienz der Rotatorenmanschette mit Dekompensation des Humeruskopfs nach kranial und entsprechende Beschwerdesymptomatik und Funktionsdefizit.

Die Durchführung der Wechseloperationen findet in der Regel in einer modifizierten Beach-Chair-Position mit ca. $30-40^{\circ}$ Oberkörpererhöhung statt. Der Patient muss weit außen gelagert und fixiert werden, damit das Schultergelenk entsprechend exponiert werden kann. Auf einem Schultertisch kann der Arm gelagert werden.

In dieser Situation empfiehlt sich auf jeden Fall der vordere deltoideopektorale Zugang, um diesen auf den Schaft von ventral zu erweitern. Die Darstellung des proximalen Humerus gestaltet sich häufig schwierig, da ausgedehnte Vernarbungen vorliegen. Diese müssen reseziert, eine subakromiale Arthrolyse und auch in der Regel eine Tenodese der Bizepssehne durchgeführt werden. Dann erfolgt die Präparation des Schaftes ventral bei Neutralrotation des Armes in Verlängerung des Sulcus intertubercularis unter Erhalt des Pectoralis-major-Ansatzes. Alternativ kann man den Pectoralis- und Deltamuskel auch ablösen und dann wieder refixieren. Der Deltamuskel wird vorsichtig lateral abgelöst unter sorgfältiger Schonung des N. axillaris.

Ein ventrales Fenster, wie von Sperling u. Cofield [7] sowie von Gohlke u. Rolf [3] beschrieben, wird mit der oszillierenden Säge angelegt. Hilfreich ist es, die Prothesenspitze bzw. Zementspitze vorher mit einem Kirschner-Draht unter radiologischer Kontrolle zu markieren, damit man weiß, wie weit nach distal die Osteotomie durchgeführt werden muss. Die Prothese bzw. der ehemalige Zementköcher wird entfernt. Ausmessen der Prothese und schließlich Verschließen des Fensters mit Drahtcerclagen, die stabiler sind als Fadencerclagen. Dabei ist sowohl auf den N. axillaris als auch auf den N. radialis weiter distal am Schaft zu achten.

Prinzipiell sollten ausreichend lange Prothesen zementfrei oder zementiert verwendet werden.

die Pfanne nach entsprechender Präparation einzementiert werden.

\section{Frakturprothese auf inverse Prothese mit Schaftwechsel (Abb. 3)}

Diese stellen für den Unfallchirurgen die häufigsten Wechseloperationen dar. Ursache hierfür sind in erster Linie die 


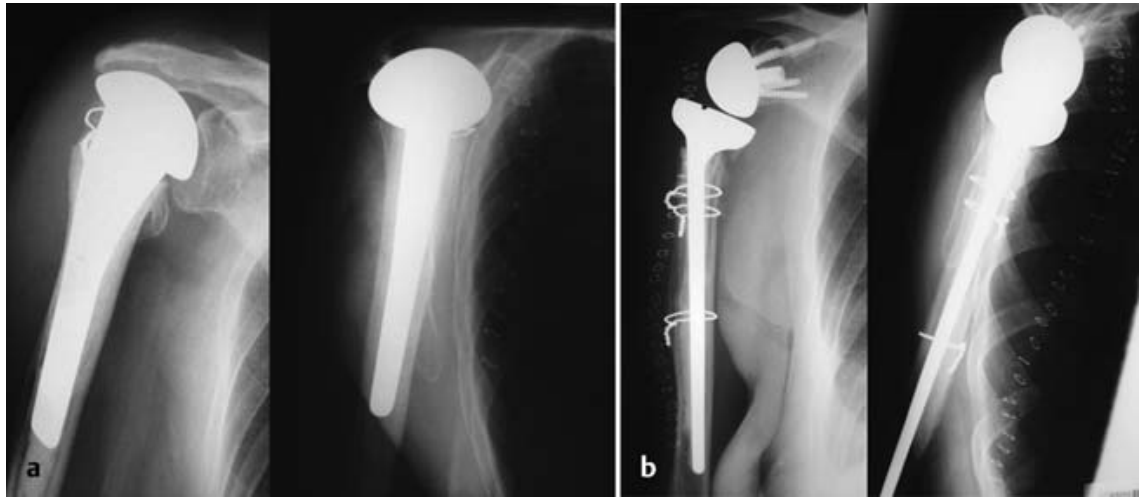

Abb. 3 a und b 71 Jahre weiblich, Röntgen a.-p. und Outlet View. a Nach kranial dekompensierte zementierte Frakturprothese mit Arrosion des Akromions und Lyse der Tubercula. b Wechsel auf eine zementierte inverse Revisionslangschaftprothese nach „Fensterosteotomie“ des Schaftes und Verschluss mit 3 Cerclagen.

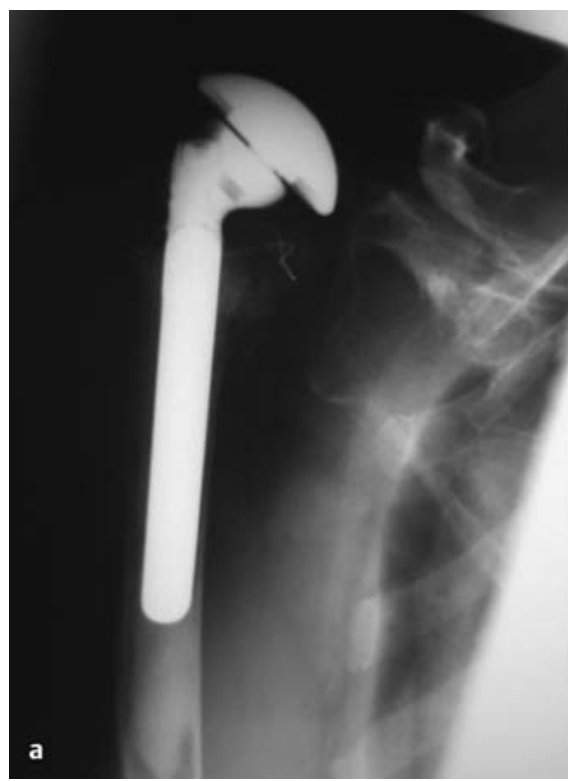

Abb.4a und b 77 Jahre weiblich, Röntgen a.-p. a Nach kranial dekompensierte zementierte modulare Frakturprothese. Nicht mehr nachweisbare Rotatorenmanschette. b Wechsel auf eine inverse Porthese bei liegendem Schaft.

\section{Frakturprothese auf inverse Prothese mit liegendem Schaft (Abb. 4)}

Prinzipiell stellt dies eine gute Alternative in der Wechseloption dar bei Prothesensystemen, die die Möglichkeit besitzen, von einer Humeruskopfprothese auf eine inverse ohne Entfernung des Schaftes zu wechseln. Das Problem besteht bei dekompensierten Frakturprothesen, die subakromial bzw. nach ventral subluxiert sind, da eine ausgedehnte muskuläre Verkürzung vorliegt und man den Schaft nicht ausreichend weit für eine inverse Prothese distalisieren kann. Nur mit einer ausgedehnten subakromialen und periprothetischen Arthrolyse kann man den Schaft nach „unten bringen“, um die Pfanne auf dem Schaft

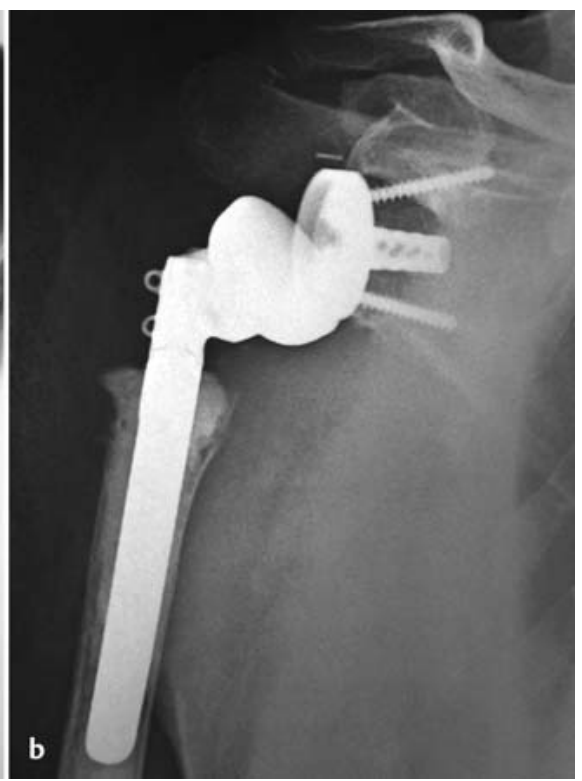

Die Wechseloption kann sich in Abhängigkeit von der Ursache einfach oder schwierig gestalten. Häufig liegt eine zu geringe Vorspannung des $\mathrm{M}$. deltoideus vor, sodass mit einer Schaftverlängerung, die durch entsprechende Aufsätze möglich ist, das Ziel einer stabilen inversen Prothese erzielt werden kann. Ein retentives Inlay bietet zusätzlich Stabilität. Handelt es sich um eine Fehlimplantation, z.B. eine Fehlrotation, oder eine deutlich pathologische Humerusschaftverlängerung bzw. auch -verkürzung, muss ein Schaftwechsel in oben beschriebener Weise durchgeführt werden.

\section{Periprothetische Frakturen}

Bei den periprothetischen Frakturen in der Schulterendoprothetik wird in der Regel eine Osteosynthese durchgeführt, da die meisten Frakturen sich in der Spitze bzw. im distalen Schaftbereich befinden und sich spiralförmig darstellen. Ein ventraler bzw. dorsaler Schaftzugang mit entsprechender Darstellung des N. radialis ist dann erforderlich. Die neuen Plattensysteme mit winkelstabilen Schrauben-Platten-Verbindungen und Polyaxialität bieten in diesen Situationen eine gute Osteosynthesemöglichkeit. Häufig müssen zusätzliche Drahtcerclagen durchgeführt werden.

Aufgrund der Zunahme der Schulterendoprothetik auch im hohen Alter nimmt auch die Anzahl der periprothetischen Frakturen bei liegender Humerusschaftprothese zu.

Sollte eine osteosynthetische Versorgung nicht möglich ein, so ist eine Revisionsendoprothetik mit Schaftwechsel in oben beschriebener Weise erforderlich. In diesen Fällen müssen Langschäfte verwendet werden, ggf. Custom-madeImplantate mit distaler Verriegelung. auch Anteile des M. pectoralis bzw. des M. latissimus dorsi im dorsalen Bereich des prxoimalen Humerusschafts hierzu ablösen. Bei nur gering dekompensierten Humeruskopfprothesen ist dies eine sicherlich atraumatische und eine elegante Wechseloption, da man den Schaft nicht osteotomieren muss.

\section{Instabile bzw. luxierte} inverse Schulterprothese

Wenn inverse Prothesen in der frühen postoperativen Phase luxieren, sind die Ursachen entweder in einer Fehlimplantation oder in einer zu geringen Vorspannung des M. deltoideus zu finden.

\section{Low-Grade oder exazerbierte Protheseninfektsituationen}

Hierbei gelten die Regeln des septischen Prothesenwechsels.

In der Regel ist ein zweizeitiges Vorgehen in Abhängigkeit von einem nachgewiesenen Keim zu propagieren.

Intermittierend sollte ein Spacer als Platzhalter implantiert werden. Dieser kann aus Palacos-Zement geformt werden und evtl. auch die spezifischen Antibiotika enthalten. Einzeitige Prothesenwechsel sind prinzipiell möglich. Dies 
kann in Ausnahmesituationen bei Verdacht auf Low-Grade-Infektion oder aseptische Prothesenlockerungen indiziert sein.

\section{Literatur}

${ }^{1}$ Aldinger PR, Raiss P, Rickert $M$ et al. Complications in shoulder arthroplasty: an analysis of 485 cases. Int Orthop 2010; 34: 517-524

2 Flury MP, Frey P, Goldhahn J et al. Reverse shoulder arthroplasty as a salvage procedure for failed conventional shoulder replacement due to cuff failure - midterm results. Int Orthop 2010; DOI: 01.1007/s00264-010-0880-Z

${ }^{3}$ Gohlke F, Rolf O. Wechsel fehlgeschlagener Frakturprothesen auf inverse Revisionsimplantate über den transhumeralen Zugang. Oper Orthop Traumatol 2007; 2: 185-207
${ }^{4}$ Pape G, Raiss P, Aldinger PR et al. Die CUPProthese mit einer zementierten Schultergelenkpfanne im Vergleich zur konventionellen anatomischen Totalendoprothese - eine Matched-Pair-Analyse. Z Orthop Unfall 2009 (Epub ahead of print)

5 Petersen SA, Hawkins RJ. Revision of failed total shoulder arthroplasty. Orthop Clin North Am 1998; 29: 519-533

${ }^{6}$ Rickert M, Loew M. Humeruskopfersatz oder Totalendoprothese bei Omarthrose? Orthopäde 2007; 36: 1013-1016

7 Sperling JW, Cofield, RH. Humeral windows in revision shoulder arthroplasty. J Shoulder Elbow Surg 2005; 14: 258-263

${ }^{8}$ Weber P, Utzschneider S, Sadoghi P et al. Management of the infected shoulder prosthesis: a retrospective analysis and review of the literature. Int Orthop 2010; DOI: 10.1007/ s00264-010-1019-3
Prof. Dr. med. Helmut Lill

Chefarzt und Ärztlicher Direktor

Dr. med. Alexandra Hertel

Fachärztin

Dr. med. Jan Christoph Katthagen

Assistenzarzt

Priv.-Doz. Dr. med. Christine Voigt

Ltd. Ärztin

Klinik für Unfall- und

Wiederherstellungschirurgie

Abteilung des Berufsgenossenschaft-

lichen Unfallkrankenhauses Hamburg

Diakoniekrankenhaus Friederikenstift

gGmbH

Humboldtstraße 5

30169 Hannover

helmut.lill@ddh-gruppe.de 